Collection SFN 11 (2010) 177-197

(C) Owned by the authors, published by EDP Sciences, 2010

DOI: $10.1051 / \mathrm{sfn} / 201011011$

\title{
Membranes pour piles à combustible : structure et transport. Apport de la diffusion neutronique
}

\author{
S. Lyonnard
}

Laboratoire des Polymères Conducteurs loniques, INACISPRAM, CEA, 17 Av. des Martyrs, 38000 Grenoble, France

\begin{abstract}
Membranes for fuel cells are characterized by a nanophase segregation between hydrophilic and hydrophobic domains. Improvement of the ionomer membranes performances, in particular protonic conductivity optimization, is a crucial issue before fuel cells can develop on an industrial market. This necessarily requires a microscopic knowledge of the polymer structure and properties, notably the protonic transport mechanisms, as a function of its hydration state. We show the interest of neutron scattering techniques in these systems. Small-angle neutron scattering has been recently used to measure the transverse water profiles in membranes during fuel cell operation. Quasielastic neutron scattering allowed to study the dynamical properties of water in the Nafion, which is the actual reference material, and to propose a molecular scenario for the mobility of hydration protons.

Résumé. Les membranes pour piles à combustibles sont des matériaux caractérisés par une ségrégation de phase hydrophobe-hydrophile à l'échelle nanométrique. L'amélioration des performances de ces membranes ionomères, en particulier l'optimisation de la conductivité protonique, est un enjeu important pour la mise sur le marché des piles à l'échelle industrielle. Elle passe nécessairement par une caractérisation microscopique de la structuration du polymère en fonction de son hydratation, et de ses propriétés, notamment les propriétés de transport. Nous montrons l'intérêt des techniques de la diffusion des neutrons dans ces systèmes. La diffusion des neutrons aux petits angles a été employée récemment pour déterminer les profils de concentration d'eau à travers une membrane au cours du fonctionnement d'une pile. La diffusion quasiélastique a permis, quant à elle, d'étudier les mécanismes de diffusion des molécules d'eau et de préciser un scénario de la mobilité moléculaire dans le Nafion, membrane de référence.
\end{abstract}

\section{INTRODUCTION}

Nous serons plus de 10 milliards sur la planète en 2050. La consommation d'énergie mondiale ne cesse de croître depuis le XIX ${ }^{\mathrm{eme}}$ Siècle : 3 Gtep $^{1}$ en 1950, 10 Gtep en 2000, et demain ?

Bien qu'il soit difficile d'établir un pronostic fiable, toutes les études, des plus optimistes aux plus catastrophiques, s'accordent à prédire un fort accroissement dans un avenir proche. On consommera en 2100 entre 20 et 50 Gtep. Or la consommation énergétique mondiale repose à $80 \%$ sur les trois grandes énergies fossiles : pétrole, gaz et charbon. Ces ressources finies ne tarderont pas à s'épuiser (Table 1).

Par ailleurs, la pollution atmosphérique (pluies acides, pollutions photochimiques, diminution de la couche d'ozone, effet de serre) pose un problème écologique majeur (Figure 1).

\subsection{La pile à combustible}

Dans ce contexte où la recherche d'énergies renouvelables non polluantes est devenue une nécessité, la pile à combustible est une technologie prometteuse. Il s'agit en effet d'un système électrochimique

This is an Open Access article distributed under the terms of the Creative Commons Attribution-Noncommercial License 3.0, which permits unrestricted use, distribution, and reproduction in any noncommercial medium, provided the original work is properly cited.

${ }^{1}$ Gtep (1 Giga tonne équivalent pétrole) correspond à 1.5 gigatonnes de charbon, soit 3 gigatonnes de lignite. 
Table 1. Réserves et production de combustibles fossiles dans le Monde. Source : CEA/DES -SEE d'après BPAmmoco Statistical Review.

\begin{tabular}{|l|c|c|c|}
\hline & Réserves prouvées fin 1998 & Production 1998 & Durée de vie statique \\
\hline Pétrole & $143.4 \mathrm{Gtep}$ & $3518.9 \mathrm{Mtep}$ & 40.8 ans \\
Gaz & $144.39 \mathrm{Tm} 3$ & $2272 \mathrm{Gm} 3$ & 63.6 ans \\
Charbon, lignite, tourbe & $497 \mathrm{Gtep}$ & $2321 \mathrm{Mtep}$ & 223 ans \\
\hline
\end{tabular}
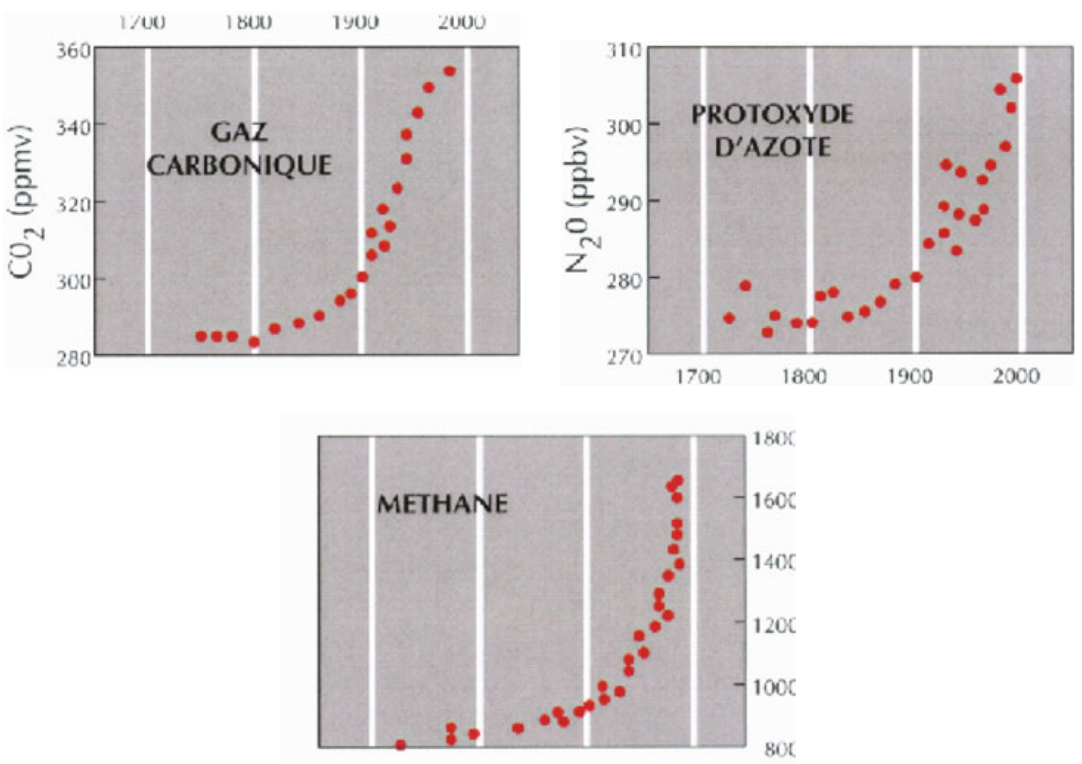

Figure 1. Production mondiale de gaz à effets de serre.

«propre» et efficace (le rendement est nettement supérieur à celui d'un cycle de Carnot). Le principe général en est simple : un électrolyte conducteur ionique sépare deux électrodes. L'anode est alimentée par un combustible, le plus souvent de l'hydrogène, la cathode par un oxydant, l'oxygène. L'énergie des réactions d'oxydation et de réduction catalytiques est transformée en énergie électrique et en chaleur. Les ions transférés par l'électrolyte sont généralement des protons $\mathrm{H}^{+}$produits à l'anode par l'oxydation du combustible, mais on trouve également des systèmes anioniques, dans lesquels les ions $\mathrm{OH}^{-}$produits à la cathode sont transférés à l'anode.

Le principe de la pile à combustible à été découvert en 1839 par Sir William Grove, qui, plongeant des électrodes de Platine dans de l'eau acidulée, observe un effet inverse à celui de l'électrolyse de l'eau. Il faut ensuite attendre plus d'un siècle pour que l'on mette en pratique cette découverte. En 1959, le tracteur Allis-Chalmers, fabriqué par le Dr. Harry K. Ihrig, est équipé d'une pile constituée de 1008 cellules et 112 empilements, capable de délivrer une puissance de $15 \mathrm{~kW}$. En 1960, un module est embarqué dans la mission spatiale Gemini : 20 minutes de fonctionnement pour un contrat de 9 millions de dollars entre la Nasa et General Electrics. A ces applications pionnières succède une longue période où la pile à combustible semble tomber dans l'oubli, avant que les considérations énergétiques et écologiques modernes ne réactivent l'intérêt des industries et de la communauté scientifique. Les piles à combustible équipent aujourd'hui des installations stationnaires de faible et moyenne puissance (stations relais en zone éloignée), des appareils portables (téléphones et ordinateurs), et toutes sortes de véhicules : du sous-marin au vélo, avion, camion, bus et, évidemment, des voitures. Dans le domaine $\mathrm{du}$ transport - terrestre, nautique et aérien - les enjeux économiques et industriels sont colossaux. Toutes les grandes marques font rouler des véhicules «propres», soit totalement alimentés par une 


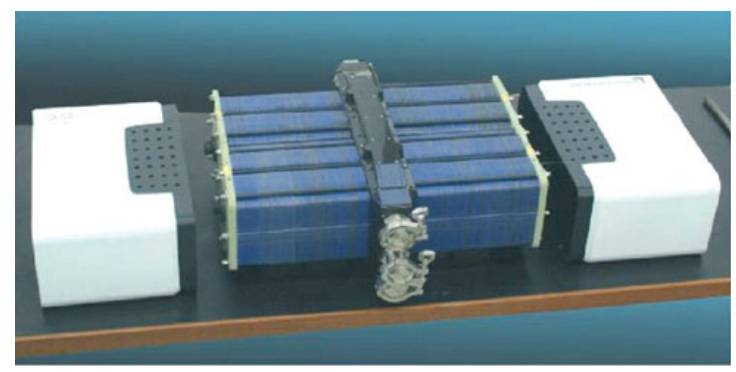

Figure 2. Le module Genepac Fuel Cell fabriqué par PSA et le CEA délivre une puissance de $4 \times 20 \mathrm{~kW}$, soit $1.5 \mathrm{~kW} / 1$ et $1 \mathrm{~kW} / \mathrm{kg}$.

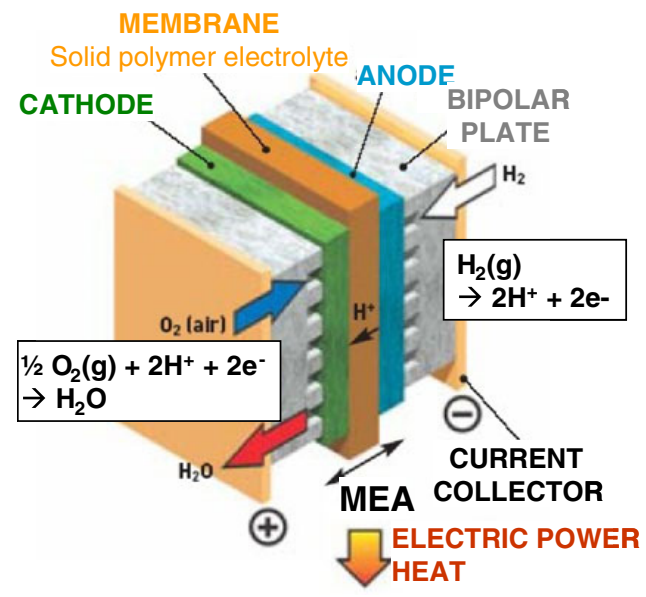

Figure 3. Schéma d'une PEMFC.

pile à hydrogène, soit hybrides. Toutefois, l'industrialisation massive tarde. Des verrous technologiques limitent encore le développement des piles en dehors de niches ou de systèmes de démonstration : gestion de la filière hydrogène (production, stockage, réseau de distribution) sans production de gaz à effet de serre (notamment sans $\mathrm{CO}_{2}$ ), optimisation de l'assemblage système, optimisation des coûts et des performances de la pile elle-même.

Il existe une grande variété de piles à combustible : les piles à électrolyte solide, qui contiennent un électrolyte à base d'Oxyde de Zirconium ou de Perovskite et sont étudiées pour les applications stationnaires (température de fonctionnement entre $900^{\circ}$ et $1000^{\circ} \mathrm{C}$ ), les piles à carbonate fondus $\left(\mathrm{Li}_{2} \mathrm{CO}_{3} / \mathrm{K}_{2} \mathrm{CO}_{3}\right)$, qui fonctionnent autour de $650^{\circ} \mathrm{C}$ et ont l'avantage de pouvoir reformer le combustible in situ (le méthane en général), les piles à acide phosphorique fonctionnant autour de $200^{\circ} \mathrm{C}$ (l'electrolyte est constitué d'acide phosphorique liquide), les piles alcalines qui fonctionnent à $80^{\circ} \mathrm{C}$ et dont l'électrolyte est une solution de potasse concentrée, et enfin les piles à membrane polymères $\left(\mathrm{PEMFC}^{2}\right.$ ) qui fonctionnent à basse température (environ $80^{\circ} \mathrm{C}$ ) et utilisent une membrane ionomère comme électrolyte.

Les PEMFC sont les piles à combustibles les plus étudiées à l'heure actuelle du fait de leurs nombreuses applications potentielles : ce sont elles qui équipent les prototypes développés par les grands constructeurs automobiles.

\footnotetext{
2 Proton Exchange Fuel Cell.
} 
a)

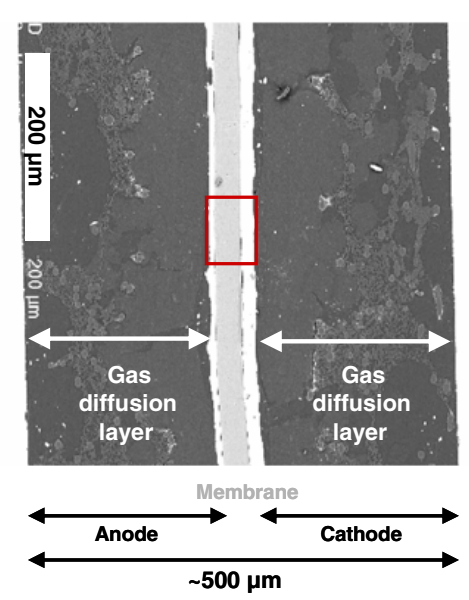

b)

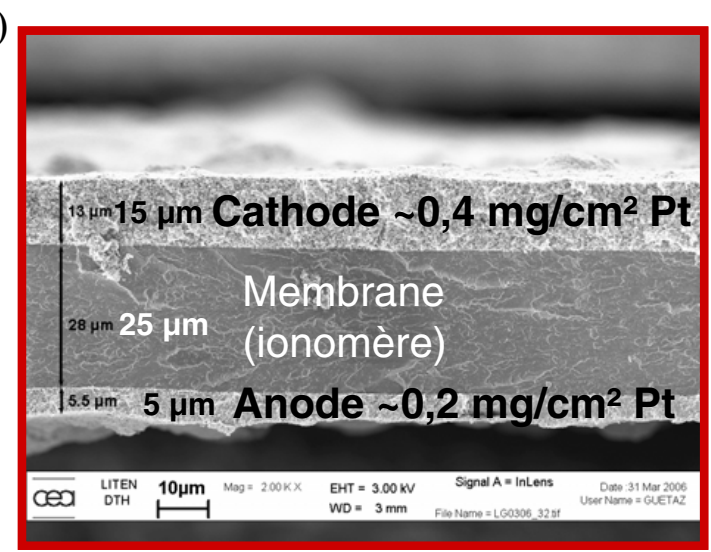

Figure 4. Images par Microscopie Electronique. a) AME complète. b) Section transversale.

Le cœur de pile, appelé AME (Assemblage Membrane Electrodes), est l'élément central du dispositif. Un effort considérable est mis en place ces dernières années pour optimiser les performances, notamment la conductivité protonique, la durée de vie du système, la température de fonctionnement et les coûts de fabrication. L'AME est un empilement de couches avec des fonctions différentes : la couche de diffusion permet l'arrivée des gaz, la couche active contient les nanoparticules de Pt catalysant les réactions électrochimiques, et enfin la membrane. Chacune de ces couches fait l'objet d'études spécifiques, et l'assemblage lui-même doit être optimisé.

\subsection{La membrane ionomère}

La membrane joue le rôle de séparateur de gaz et de conducteur protonique. De manière générale, et en plus des propriétés essentielles de conduction ionique et de stabilité chimique, les membranes ionomères pour PEMFC doivent remplir un cahier des charges très contraignant : bonnes propriétés mécaniques sous contrainte, température de transition vitreuse élevée (afin d'augmenter la stabilité mécanique en température), propriétés «barrières » à l'oxygène et à l'hydrogène, conductivité électronique nulle, coûts de synthèse et de fabrication raisonnables (une voiture embarque typiquement de l'ordre de $10 \mathrm{~m}^{2}$ de membrane).

Actuellement, la membrane de référence pour la PEMFC, en terme de performances et de durée de vie, est le Nafion $^{3}$. Il s'agit d'un polymère perfluoré sulfoné composé d'une chaîne fluoro-carbonée (type Téflon ${ }^{\circledR}$ ) sur laquelle sont greffées des chaînes pendantes (perfluorovinyléther) terminées par un groupe ionique sulfonate. Initialement conçu comme séparateur pour l'électrolyse chlore/soude [1], il est utilisé dans la majorité des systèmes commercialisés fonctionnant aujourd'hui. Un tel monopole vient de ses propriétés remarquables, notamment : une très grande stabilité chimique à la réduction et à l'oxydation; une grande stabilité chimique à l'hydrolyse en milieu acide; une conductivité ionique élevée (de l'ordre de $10^{-1} \mathrm{~S} / \mathrm{cm}$ dans les meilleures conditions [2]). De plus, il peut être mis en solution, ce qui est un point important pour la fabrication des électrodes pour PEMFC.

Cependant, le Nafion possède aussi un inconvénient majeur : c'est un matériau qui gonfle beaucoup, typiquement de $15 \%$ entre l'état sec et l'état complètement hydraté. Lors d'un fonctionnement en stationnaire, ceci ne pose pas de problèmes particuliers (bonne durée de vie, 50000 heures mesurées [3]).

${ }^{3}$ Le Nafion ${ }^{\circledR}$ est une marque déposée de la société Du Pont de Nemours. 


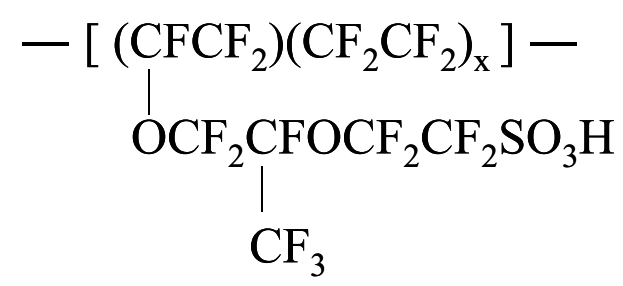

En revanche, dès que la pile fonctionne en cycles démarrages/arrêts, la membrane Nafion subit une alternance de gonflements/contractions. Ce cyclage entraîne des variations dimensionnelles répétées et génère un stress mécanique important : la membrane devenue fragile peut se percer rapidement, la pile cesse de fonctionner. D'autre part, le Nafion est un polymère de basse $\mathrm{T}_{g}$ (température de transition vitreuse), ce qui pose des problèmes de tenue mécanique et d'efficacité dès que l'on excède les $80^{\circ} \mathrm{C}$. A ces problèmes technologiques s'ajoute un prix prohibitif : équiper une voiture d'un moteur électrique alimenté par une PEMFC au Nafion revient à près de $8000 \breve{A}$ rien que pour l'achat de la membrane!

Par conséquent, la recherche de matériaux alternatifs au Nafion est devenue nécessaire. Bon nombre de travaux sont menés dans le but de synthétiser des matériaux ayant des performances équivalentes, ne présentant pas les mêmes limites et pouvant être fabriqués à grande échelle et à moindre coût [4]. L'enjeu industriel est évidemment colossal.

A l'heure actuelle, plusieurs familles de matériaux existent; certaines sont commercialisées, d'autres encore sujettes à études :

- La famille des polymères perfluorés. C'est la famille dans laquelle se range le Nafion. La chaîne principale est fluorée et des chaînes pendantes, terminées par un groupe sulfonate, sont greffées sur le squelette de type PTFE. Les noms commerciaux des différents matériaux sont Flemion ${ }^{4}$, Aciplex-S ${ }^{5}$, Dow $^{6}$, etc. Ces membranes sont de structure chimique proche de celle du Nafion et ont des durées de vie comparables.

- La famille des polymères non fluorés à squelette aromatique. Dans cette vaste famille se trouvent des membranes sous forme d'acides sulfoniques (par exemple l'acide polystyrène-divinylbenzène [5]), des polymères éthers aromatiques (par exemple les poly(arylène ether sulfone) [6]), les polyether ether cétone (PEEK) [7], les polyimides sulfonés (PIs) [8], etc. Leur avantage principal est leur faible coût de synthèse.

- La famille des membranes composites. Les membranes perfluorées peuvent être renforcées pour en améliorer les propriétés mécaniques ou diminuer le gonflement, par exemple par du polytétrafluoroéthylène incorporé dans le Nafion [9] (membrane Gore-Select ${ }^{\mathrm{TM}}$ ). On peut également mélanger différents polymères, un ionomère sulfoné et un polymère non chargé typiquement. Le mélange garde les propriétés de conductivité ionique du polymère sulfoné et est renforcé par le second composé qui ne gonfle pas [10]. Une autre voie est de former un composite organique-inorganique : polymère ionique avec un «enduit» inorganique comme support mécanique, polymère ionique avec un «enduit» inorganique piégeant l'eau, additif inorganique conducteur ionique avec un support polymère, etc. Les possibilités sont nombreuses. Pour l'instant, il s'agit encore de matériaux testés en laboratoire.

\footnotetext{
${ }^{4}$ Le Flemion ${ }^{\circledR}$ est un produit de la société Asahi Glass.

5 Aciplex-S ${ }^{\circledR}$ est un produit de la société Asahi Chemical.

${ }^{6}$ Dow ${ }^{\circledR}$ est un produit de la société Dow Chemical.
} 


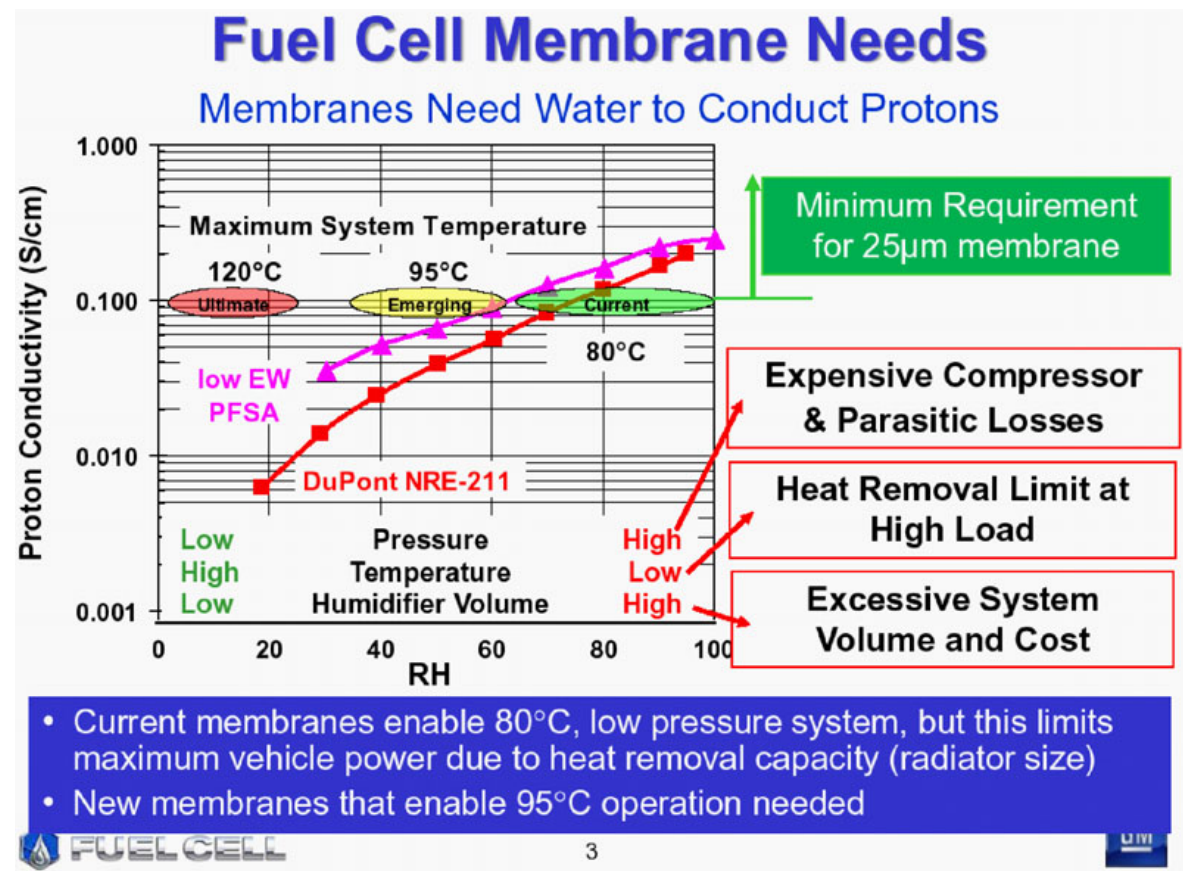

Figure 5. Les objectifs de General Motors, C, Gittleman, General Motors Fuel Cells Research Lab. Advances in Polymer Electrolyte Fuel Cell Membrane Systems, Asilomar Conference 2009.

\subsection{Optimisation des membranes et gestion de l'eau}

Quelle sera la membrane de demain ? C. Gittleman présente en 2009 les objectifs de General Motors (figure 5) : obtenir des systèmes opérant à $120^{\circ} \mathrm{C}$ et à faible humidité.

Membranes need water to conduct protons, c'est en quelque sorte la phrase clé qui détermine les stratégies d'optimisation actuelles. La gestion de la quantité d'eau présente dans l'électrolyte au cours $\mathrm{du}$ fonctionnement d'une pile est en effet une question centrale. D'une part, la conductivité dépend de l'état d'hydratation de la membrane (Figure 6). L'eau, vecteur du transport des protons, pilote la capacité d'un film à transférer efficacement les charges de l'anode à la cathode. D'autre part, au cours du fonctionnement d'une pile, l'état d'hydratation de la membrane varie au cours du temps et suivant les conditions opératoires (température, densité de courant, etc.). Les risques d'assèchement ou au contraire de noyade constituent une limitation technologique importante.

Deux questions fondamentales se posent :

Où est l'eau?

- Quel est son rôle? Quelles sont ses propriétés?

La distribution d'eau dans une AME est en effet non uniforme (Figure 7). Dans l'épaisseur de la membrane, un gradient de concentration résulte de la compétition entre les phénomènes d'électroosmose (transport de molécules d'eau par le courant protonique) et de rétrodiffusion (la réduction de l'oxygène produit de l'eau à la cathode, d'où la diffusion des molécules de la cathode à l'anode sous l'effet de gradients d'activité). De plus, la répartition de l'eau à la surface de l'AME, entre le point d'entrée des gaz humidifiés et la sortie, est également très inhomogène.

Enfin, l'origine moléculaire des mécanismes du transport protonique, étroitement liés à l'état d'hydratation de la membrane, est encore méconnue.

Répondre à ces deux questions, où est l'eau, à quoi sert-elle, est une nécessité pour comprendre les performances et la durée de vie des membranes et plus généralement des AMEs. Mais comment 


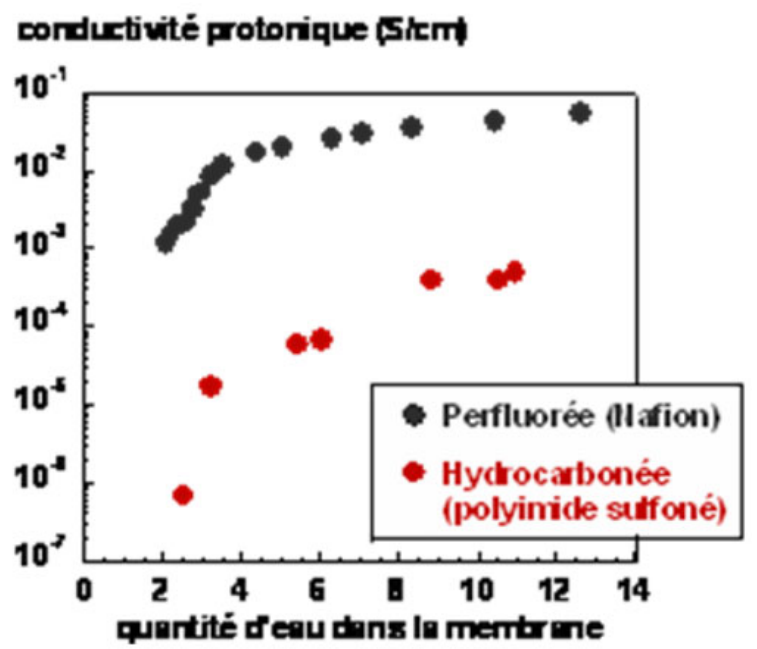

Figure 6. Conductivité protonique du Nafion en fonction de la quantité d'eau dans la membrane (nombre de molécules d'eau par site ionique), comparée à celle d'un concurrent polyaromatique, le polyimide sulfoné.
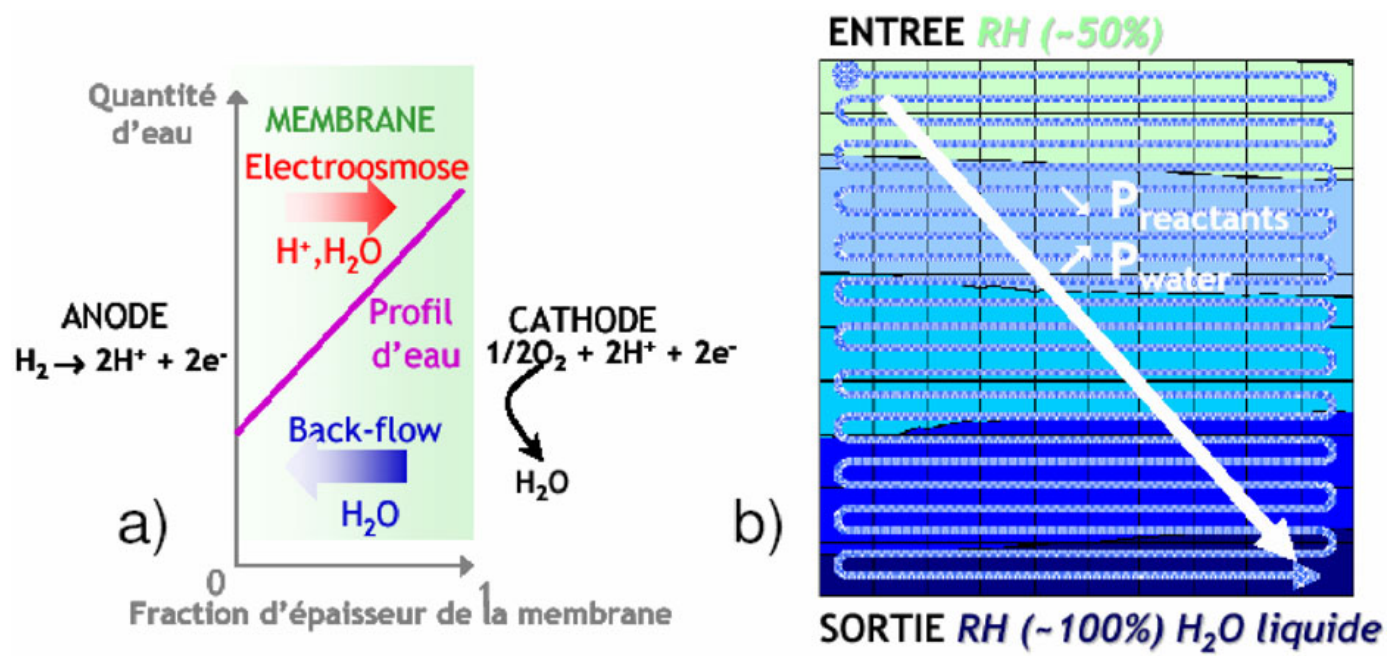

Figure 7. Distribution d'eau a) dans l'épaisseur de la membrane b) à la surface de l'AME.

quantifier la distribution d'eau dans une membrane entre les électrodes pendant un fonctionnement standard sans être intrusif ? Et comment mesurer la mobilité de l'eau à l'échelle moléculaire et évaluer l'effet du confinement dans la membrane?

Avec les neutrons ! Nous présentons dans la suite l'intérêt d'expériences de Diffusion des Neutrons aux Petits Angles (DNPA) et de diffusion quasi-élastique des neutrons (QENS) appliqués à la problématique de l'eau dans la membrane.

\section{DIFFUSION DE NEUTRONS AUX PETITS ANGLES : UNE PILE DANS LE FAISCEAU}

La diffusion des neutrons aux petits angles (DNPA) est une technique de choix pour étudier la nanostructuration des systèmes complexes. Une expérience originale a été réalisée récemment au 


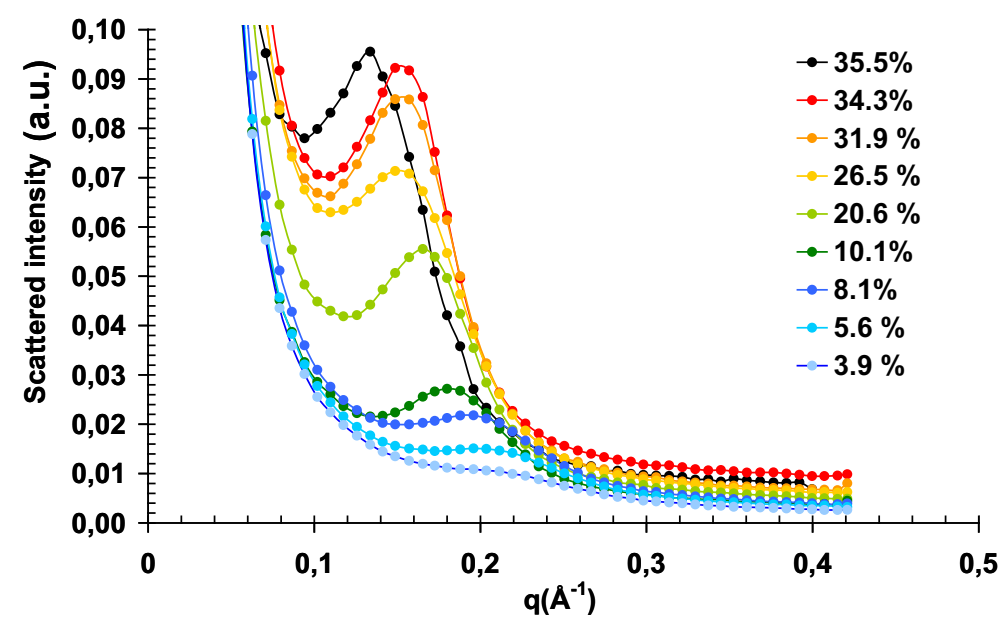

Figure 8. Spectres de diffusion des neutrons aux petits angles obtenus sur des membranes Nafion à différents taux de gonflement massique. Le gonflement est imposé par le choix d'une solution saline fixant la pression de vapeur saturante et donc l'humidité relative dans la cellule contenant la membrane.

Laboratoire Léon Brillouin (sur le spectromètre PAXE) en mettant une pile à combustible spécialement conçue à cet effet dans un faisceau de neutrons [11, 12]. L'analyse des spectres de diffusion des neutrons en fonction du vecteur de diffusion Q permet d'établir les profils d'eau dans l'épaisseur de la membrane en fonction des paramètres opératoires du système.

\subsection{Structure du Nafion à l'équilibre}

Le Nafion [13] est caractérisé par une nanoséparation de phases entre domaines hydrophiles et hydrophobes, propriété qui lui confère sa capacité de conducteur protonique. Les protons dissociés de leurs groupements $\mathrm{SO}_{3}^{-}$diffusent dans un réseau de pores interconnectés. Expérimentalement, cette ségrégation de phase se traduit par l'existence d'un pic appelé «pic ionomère» dans le spectre de diffusion des neutrons aux petits angles. La position du pic est reliée à la distance caractéristique interdomaines ioniques, typiquement de 20 à $50 \AA$, tandis que son intensité, du fait de l'hydrophobicité du squelette fluoré, résulte directement du contraste entre domaines hydrophiles et hydrophobes. Plusieurs modèles concurrents sont proposés pour décrire la structuration multi-échelle complexe du Nafion. Une organisation des chaînes hydrophobes en agrégats allongés plongés dans un continuum ionique a été récemment proposée $[14,15]$. Les agrégats s'organisent en fagots d'une taille typique $>800 \AA$.

Dans les ionomères en général et le Nafion en particulier, la structure dépend directement de la quantité d'eau dans le matériau. Par diffusion des neutrons, on observe le gonflement de la membrane via la déplacement du pic ionomère aux petits angles et l'augmentation de l'intensité de ce pic (Figure 8). La DNPA sonde ainsi directement la quantité d'eau moyenne dans l'échantillon.

\subsection{Méthode d'analyse : comment obtenir des profils d'eau}

On sait que, dans la membrane sous tension dans la pile, il existe un profil d'eau, avec une accumulation à la cathode et un assèchement à l'anode (Figure 9(a)). On peut découper la membrane dans son épaisseur en tranches élémentaires (Figure 9(b)), chaque tranche $i$ étant caractérisée par une hydratation moyenne constante $w_{i}$ (taux de gonflement massique).

Les spectres de diffusion correspondant aux valeurs moyennes $\mathrm{w}_{i}=3.9,5.6,8.1,10.1,20.6,26.5$, $31.9,34.3$ et $35.5 \%$ ont été mesurés sur des membranes à l'équilibre (Figure 8). N'importe quel profil 


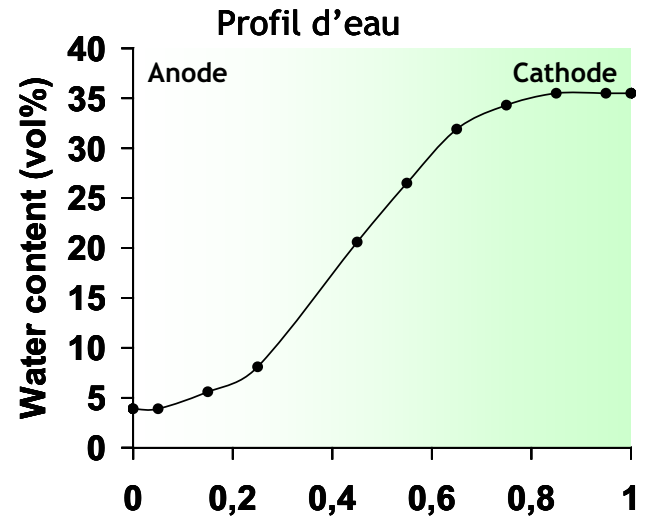

Fraction of membrane thickness

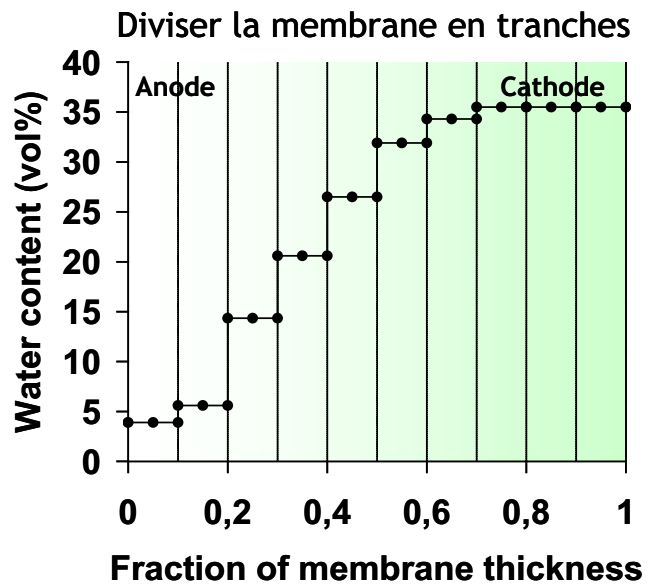

Figure 9. a) Profil d'eau dans une membrane et b) découpage en tranches.
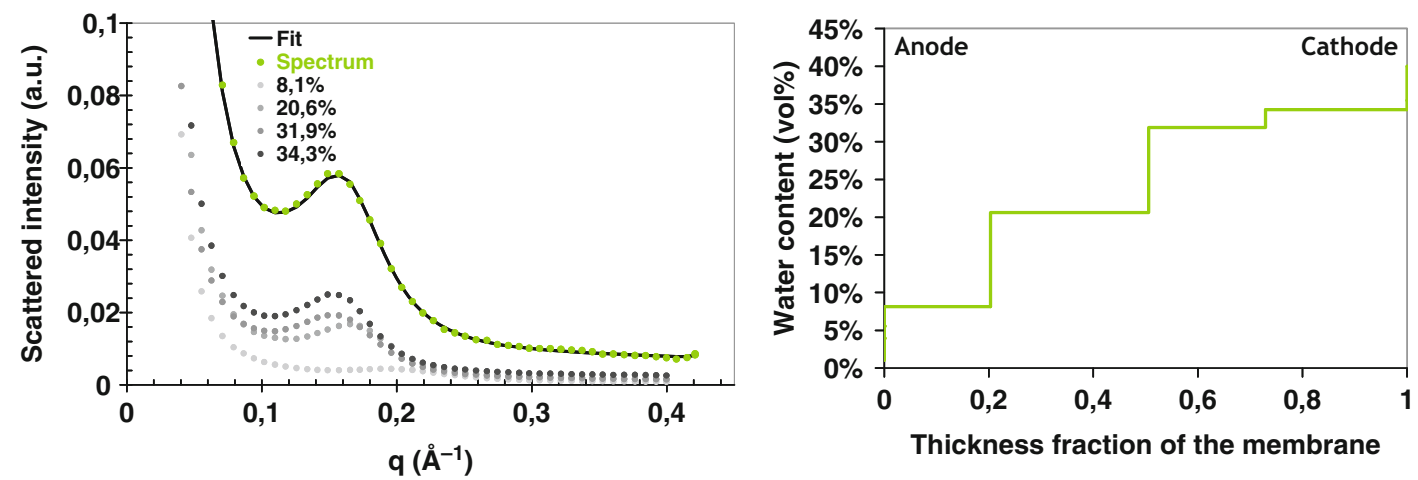

Figure 10. a) Ajustement d'un spectre de diffusion donné avec une combinaison de quatre spectres d'hydratation de référence, à 8.1, 20.6, 31.9 et $34.3 \%$. b) Profil d'eau dans la membrane correspondante. Les fractions d'épaisseur à hydratation constante sont les coefficients de la décomposition sur la base des spectres de référence.

d'eau dans la membrane pourra se décomposer sur la base des tranches constantes, et, par conséquent, le spectre de diffusion associé se décompose également sur la base des spectres de référence :

$$
\mathrm{I}(\text { total })=\Sigma \mathrm{I}(\text { tranche }) \times \mathrm{e}(\text { tranche }) .
$$

Mesurons maintenant le spectre de diffusion par DNPA d'une membrane hydratée. En faisant l'hypothèse que la quantité d'eau à la cathode est supérieure à celle à l'anode, que les profils de concentration sont monotones, et qu'il n'y a pas de diffusion multiple dans l'échantillon, on peut trouver une combinaison unique de spectres de base permettant d'ajuster le spectre (Figure 10(a)). On peut ainsi déterminer le profil d'eau dans la membrane (Figure 10(b)).

\subsection{Montage expérimental}

Une pile spéciale a été conçue pour l'expérience de DNPA. Il s'agit d'une cellule en Aluminium recouvert d'or de $25 \mathrm{~cm}^{2}$. Un seul canal en forme de serpentin permet d'acheminer les gaz à la surface des plaques monopolaires. La configuration de type co-flow a été adoptée, c'est-à-dire que l'arrivée de $1^{\prime} \mathrm{O}_{2}$ et de $\mathrm{l}^{\prime} \mathrm{H}_{2}$ se fait par le haut. Trois trous ont été percés dans le corps de la pile pour permettre de 

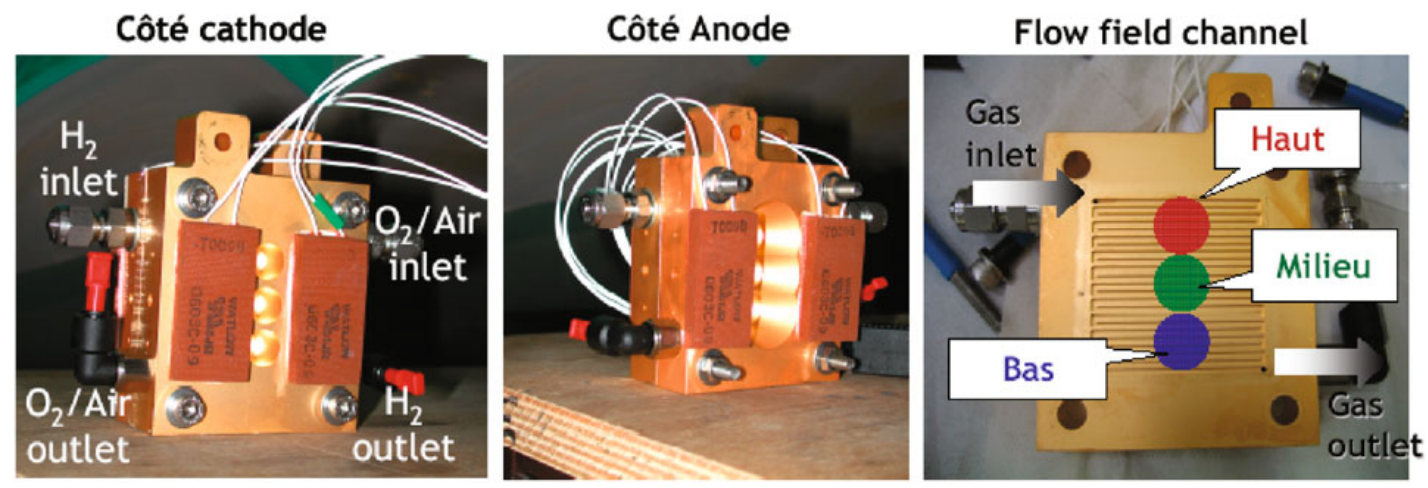

Figure 11. Pile à combustible conçue pour l'expérience de DNPA.

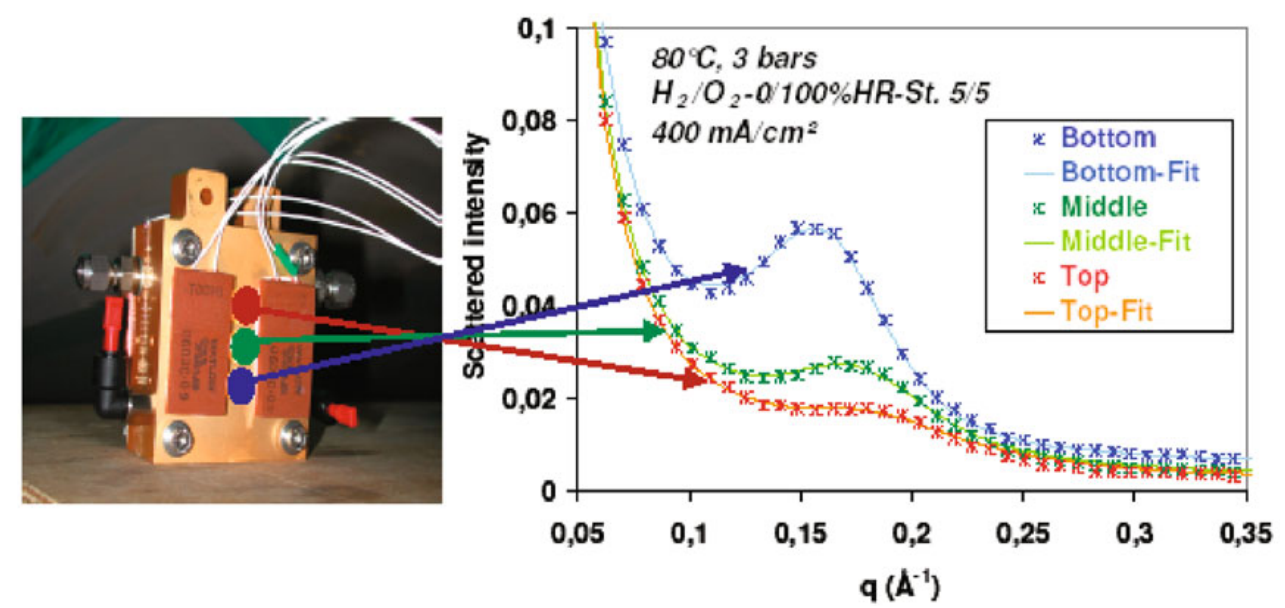

Figure 12. Spectres de DNPA enregistrés après passage du faisceau de neutrons dans les trois trous (Haut, Milieu, Bas) percés dans la pile.

sonder trois endroits différentes : le haut de la pile (arrivée des gaz), le bas de la pile (sortie des gaz), et une position intermédiaire au milieu.

Les paramètres contrôlés pendant l'acquisition des spectres de petits angles sont : la température, la pression des gaz, l'humidification des gaz, leur débit, le courant ou la tension ou la puissance.

\subsection{Résultats}

Les contributions de la cellule vide et des couches de diffusion des gaz, qui donnent notamment un fort signal aux très petits angles, ont été soustraites des spectres bruts.

\subsubsection{Inhomogénéité de la distribution d'eau}

Les spectres de diffusion obtenus sur une pile fonctionnant à $80^{\circ} \mathrm{C}$, sous 3 bars de pression, dans la stoechiométrie $\mathrm{H}_{2} / \mathrm{O}_{2} 5 / 5$ avec de $1^{\prime} \mathrm{H}_{2}$ sec et de $1^{\prime} \mathrm{O}_{2}$ à $100 \%$ d'humidification, délivrant un courant de $400 \mathrm{~mA} / \mathrm{cm}^{2}$ (ces conditions sont représentatives du fonctionnement réel) montrent que la quantité d'eau moyenne n'est pas homogène. Les trois spectres enregistrés dans les positions Haut, Milieu et Bas sont en effet très différents (Figure 12). Les profils d'eau correspondant, déduits par la 


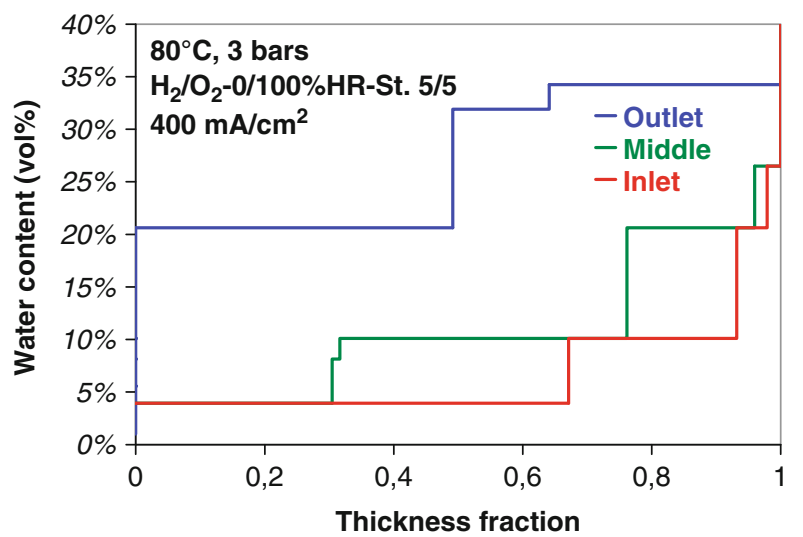

Figure 13. Le profil d'eau à la sortie de la pile (position Bas) est quasiment constant. La membrane est complètement humidifiée à la cathode, alors qu'on observe un assèchement à l'anode à cause du phénomène d'électroosmose.
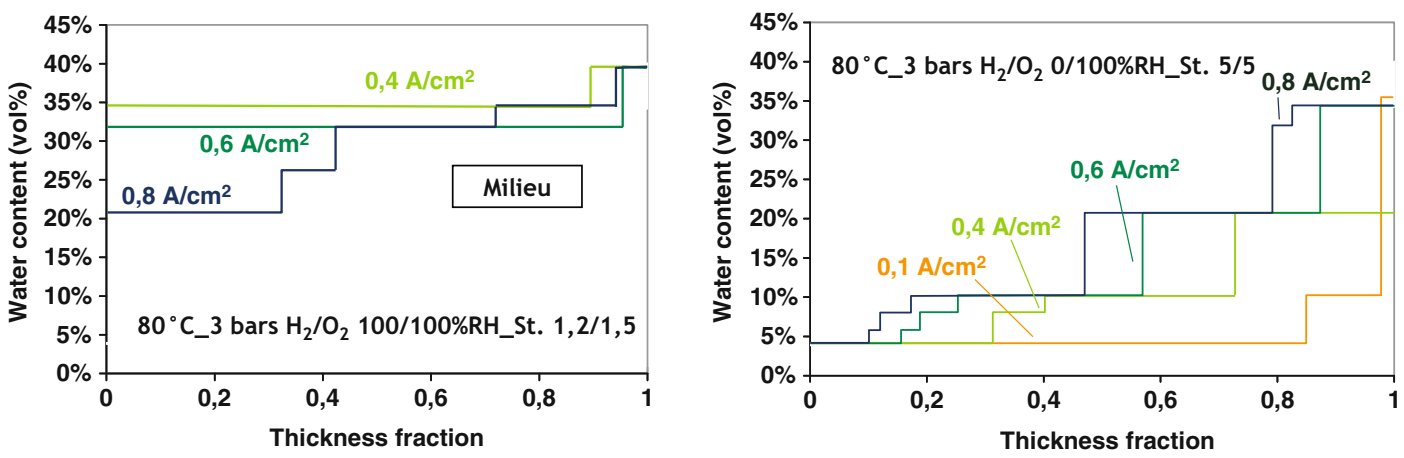

Figure 14. Profils d'eau dans l'épaisseur de la membrane pour différentes densités de courant, à $\mathrm{T}=80^{\circ} \mathrm{C}, 3$ bars de pression et $\mathrm{H}_{2} / \mathrm{O}_{2} 1.2 / 1.5$. a) Anode et cathode hautement humidifiées, faible débit de gaz. b) Anode sèche, cathode hautement humidifiée, fort débit de gaz.

méthode d'analyse présentée précédemment, montrent l'inhomogénéité de la distribution de l'eau dans l'épaisseur de la membrane (Figure 13).

\subsubsection{Compétition entre électroosmose et rétrodiffusion}

Les spectres de diffusion ont été enregistrés dans des conditions de débit et d'hydratation des gaz variables, à température constante $\left(80^{\circ} \mathrm{C}\right)$, pression et stoechiométrie constantes ( 3 bars, $\left.1.2 / 1.5\right)$, afin d'évaluer la compétition entre électrosomose et rétrodiffusion dans la membrane. On observe que lorsque les gaz en entrée à la cathode et à l'anode sont hautement humidifiés, avec un faible débit, l'augmentation de la densité de courant $\left(\mathrm{de} 0.4\right.$ à $\left.0.8 \mathrm{~A} / \mathrm{cm}^{2}\right)$ entraîne une diminution de la quantité d'eau. L'électrosomose l'emporte dans ce cas sur la rétrodiffusion. Au contraire, si le gaz est sec à l'entrée de l'anode et complètement hydraté à l'entrée de la cathode, avec un haut débit, on observe le comportement inverse : l'augmentation de la densité de courant s'accompagne d'une augmentation de la quantité d'eau dans la membrane. La rétrodiffusion prend alors le pas sur l'électroosmose. Ces résultats (figure 14) montrent que la balance entre électrosomose et rétrodiffusion des molécules d'eau au travers de la membrane dépend des conditions de fonctionnement. 


\subsubsection{Conclusions : apports de la DNPA}

La DNPA est un outil non intrusif permettant de déterminer des profils de concentration d'eau dans une membrane au cours du fonctionnement réel d'une pile à combustible. L'obtention de ces profils est extrêmement utile pour valider les modèles de transfert de charges et de masse dans les piles. La faible résolution spatiale (taille du faisceau de l'ordre de $10 \mathrm{~mm}$ ) et temporelle (acquisition d'un spectre toutes les minutes) de l'expérience pose une limitation, qui pourrait être levée en développant la méthode par Diffusion des Rayons X aux Petits Angles. L'utilisation du faisceau synchrotron permettrait ainsi d'atteindre une résolution spatiale de typiquement 300 microns, et d'effectuer des acquisitions toutes les trente secondes, voire moins.

\section{QENS : LE NAFION PLUS OU MOINS GONFLÉ DANS LE FAISCEAU}

\subsection{Le Nafion hydraté : une dynamique multi-échelle}

La membrane Nafion sépare les électrodes et assure le transfert des protons dans la pile. Le mécanisme complexe du transport protonique [16, 17] fait l'objet d'un intense effort de recherche (simulations numériques, modélisations, expériences). L'optimisation de la conductivité est en effet un enjeu majeur à l'échelle internationale pour le développement technologique et industriel des piles. Le transport protonique est intimement lié à la dynamique des molécules d'eau, d'où l'importance de caractériser les propriétés de l'eau d'hydratation aux échelles microscopiques pour proposer des scénarios moléculaires capables de décrire les réponses macroscopiques du système en terme de conductivité.

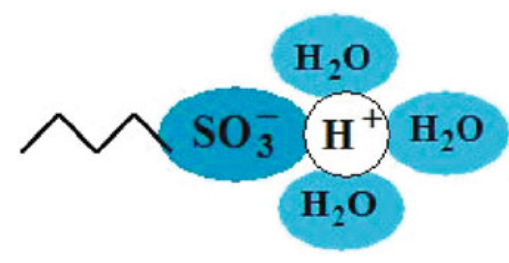

Toutes les propriétés du Nafion - et des membranes ionomères en général (conductivité [18], coefficients d'électro-osmose [19, 20], coefficients de diffusion de l'eau et du proton [21, 22], tenue mécanique, comportement en pile, tenue en température, dégradation, etc.) - ainsi que la nanostructuration de la matrice polymère et les mouvements de l'eau et du proton à toutes les échelles, dépendent de l'état de gonflement à l'équilibre. Cet état d'hydratation est caractérisé par une valeur normalisée permettant de comparer les matériaux entre eux : le nombre de molécules d'eau par site ionique, noté $\lambda$.

L'eau est confinée dans la matrice complexe. Le milieu est chargé : les parois des «pores» portent des groupements sulfoniques $\mathrm{SO}_{3}^{-} \mathrm{H}^{+}$. Le confinement nanoscopique a un effet spectaculaire sur les propriétés des fluides moléculaires en général, et de l'eau en particulier [23]. Quand l'eau est confinée dans des pores de petite taille, c'est-à-dire jusqu'à la portée des forces intermoléculaires, ses propriétés thermodynamiques, structurales et dynamiques sont profondément altérées par rapport à la situation en volume. En particulier, le confinement spatial affecte considérablement la mobilité des molécules. Lorsque l' «interface» est de surcroît chargée, les interactions entre groupes ioniques et ions ou molécules s'ajoutent à l'effet purement géométrique. Ceci se traduit par une modification des mécanismes intrinsèques de la diffusion et une variation importante des paramètres dynamiques macroscopiques, parfois sur plusieurs ordres de grandeur. La nature de l'interface fluide-matrice, sa morphologie, sa rugosité, sont déterminantes. 
Toutes les études structurales menées sur le Nafion révèlent une structure complexe et organisée à différentes échelles d'espace. Cette hiérarchie d'échelles spatiales a un impact fort sur la mobilité de l'eau et ses propriétés : la dynamique elle-aussi est «multi-échelles». Conditionnée par le confinement nanométrique mais aussi par les surstructures, elle s'étend sur une très large gamme de temps de relaxation : durée de vie d'une liaison hydrogène (typiquement la picoseconde), d'un ion hydronium ou d'un complexe hydraté, temps d'adsorption à la surface d'un pore, temps de diffusion dans un pore, d'un pore à l'autre, d'un domaine à un autre, jusqu'au temps de diffusion global dans le matériau, etc... Ces temps caractéristiques sont associés à différents mécanismes de diffusion, comme le mécanisme de sauts de type Grotthuss, le mécanisme de transport véhiculaire, le mécanisme de diffusion surfacique, qui souvent sont imbriqués ou corrélés [24, 25]. Il n'y a donc pas une seule échelle pertinente pour une étude des propriétés dynamiques. Il est fondamental de pouvoir observer le système à l'échelle très locale des mouvements moléculaires fortement affectés par la présence de l'interface, à «l'échelle micrométrique» correspondant par exemple à la diffusion inter-domaines poreux orientés, et également aux échelles «mésoscopiques» (dites intermédiaires), qui sont les plus difficiles à atteindre expérimentalement et correspondent pourtant à des phénomènes de première importance (exploration d'un pore ou d'un espace inter-lamelles par exemple).

Une méthodologie multi-échelle, basée sur la synergie entre caractérisations expérimentales et simulations numériques à toutes les échelles pertinentes, est par conséquent adaptée dans ce contexte. La spectroscopie RMN fournit des informations aux échelles mésoscopiques (par l'usage de la relaxométrie RMN qui permet d'accéder via des modèles à la gamme des temps dits «intermédiaires ») et micrométriques (mesure des coefficients d'auto-diffusion de l'eau par la technique des gradients de champs pulsés). Pour ce qui concerne l'étude locale des mouvements moléculaires, c'est la diffusion quasi-élastique des neutrons qui est la plus appropriée. Nous allons montrer ici quelles informations quantitatives peuvent être extraites par QENS et leur impact sur la compréhension globale des mécanismes du transport et de la dynamique.

\subsection{Le QENS et le Nafion : un couple qui fonctionne}

La diffusion quasi-élastique des neutrons est une technique spectroscopique adaptée à l'étude de la dynamique des ions et des molécules, notamment en situation de confinement [26]. La gamme de transfert d'énergie sondée est de quelques $\mathrm{meV}$, de l'ordre de grandeur des mouvements de relaxation ou de diffusion (translation ou rotation des molécules) dans la matière. La fonction mesurée $S(Q, \omega)$ est proportionnelle à un terme de contraste entre les atomes diffusants, déterminé par les sections efficaces de diffusion cohérentes et incohérentes des noyaux nucléaires. La diffusion cohérente (incohérente) traduit l'existence de processus collectifs (individuels). L'atome d'hydrogène est un diffuseur incohérent naturel : sa section efficace incohérente est typiquement un ordre de grandeur supérieure à celle de tous les autres atomes. Cette propriété est à l'origine de l'intérêt du QENS pour l'étude de la dynamique individuelle du proton ou de l'eau.

La membrane Nafion est un excellent échantillon pour deux raisons : (i) le contraste entre les atomes constitutifs du squelette $(\mathrm{C}, \mathrm{F})$ et l'atome d'hydrogène $\mathrm{H}\left(\mathrm{ou} \mathrm{H}^{+}\right)$est élevé (ii) la matrice confinante ne contient pas du tout de $\mathrm{H}$. Ces deux caractéristiques assurent une situation idéale d'un point de vue expérimental : on attribue sans ambiguïté la composante quasi-élastique des spectres à la dynamique individuelle du proton.

Toute la difficulté de la technique provient de son caractère indirect : la mesure est effectuée dans l'espace de Fourier. Il faut, pour obtenir les grandeurs d'intérêt - temps caractéristiques de relaxation des phénomènes dynamiques, distances de saut ou de confinement, coefficients de diffusion, nature des mouvements - bâtir un modèle de diffusion. C'est ensuite l'ajustement de la fonction de diffusion expérimentale $S(Q, \omega)_{\exp }$ avec la fonction théorique $S(Q, \omega)$ qui permet de conclure. La validation d'un modèle de diffusion plutôt qu'un autre peut être considérablement renforcée en variant au cours d'une expérience les conditions de résolution, c'est-à-dire la fenêtre temporelle de la mesure. On peut ainsi 

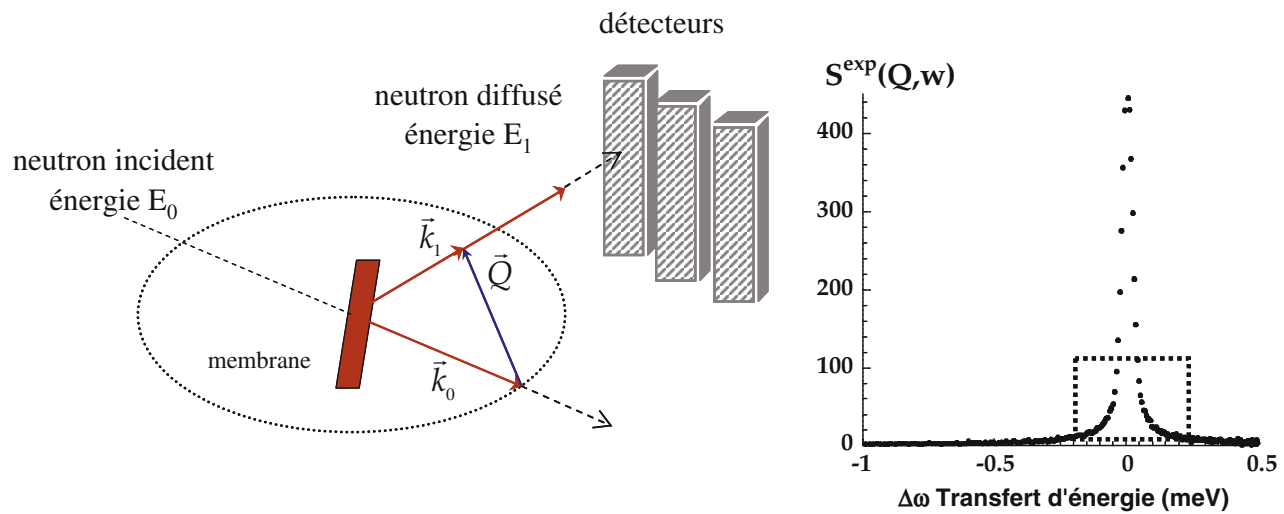

Figure 15. a) Principe d'une expérience de diffusion quasi-élastique des neutrons. Le vecteur de diffusion $\mathrm{Q}$ est défini par $\mathrm{Q}=\mathrm{k}_{1}-\mathrm{k}_{0}$ et le transfert d'énergie $\mathrm{h} \omega=\mathrm{E}_{1}-\mathrm{E}_{0}$. b) Exemple de spectre typique. Le pic central est le pic élastique, le signal quasi-élastique correspondant à de faibles transferts d'énergie est signalé par un encadré pointillé. C'est l'ajustement de ce signal par un modèle théorique de la diffusion moléculaire qui permet de remonter aux paramètres dynamiques pertinents, comme les temps de corrélation, distances caractéristiques et coefficients de diffusion.

combiner de manière fructueuse des spectromètres de temps de vol, opérant dans la gamme 1ps-100ps typiquement, et de «backscattering », dont la gamme d'observation est plutôt de l'ordre de la ns. Sur un même appareil de temps de vol, on combinera de plus des mesures effectuées à des longueurs d'onde différentes, ce qui permet de «sélectionner» différents mécanismes, pour peu qu'ils opèrent à des échelles de temps suffisamment distinctes. C'est la stratégie que nous avons employée dans le Nafion récemment [27], où il est clair que la diffusion ne saurait être traitée comme un processus dynamique unique.

\subsection{Modélisation de la diffusion confinée}

Le comportement dynamique de l'eau est fortement affecté dans le cas où elle est confinée. Les processus d'auto-diffusion des atomes $\mathrm{H}$ (diffusion translationnelle) et les réorientations de la molécule $\mathrm{H}_{2} \mathrm{O}$ (diffusion rotationnelle) sont «gênés » et en général ralentis par l'existence d'une interface, qui impose une contrainte géométrique et/ou porte des fonctions chimiques interagissant avec l'eau. La molécule d'eau ne peut plus diffuser que dans des volumes restreints. La fonction $\mathrm{S}(\mathrm{Q}, \omega)$ est donc affectée, et les modèles classiques (de type mouvement diffusif aléatoire dans un milieu infini) ne sont plus applicables. Un modèle de diffusion moléculaire adapté à la situation du confinement doit être mis en place. La fonction $\mathrm{S}(\mathrm{Q}, \omega)$ a ainsi été calculée pour différentes géométries de confinement : des modèles de diffusion sur un segment [28], dans un cercle [29], un cylindre [30] ou une sphère [30] ont été très largement utilisés pour interpréter les données obtenues sur des molécules diffusant dans des matrices poreuses, des liquides super-refroidis ou des polymères [32, 33].

Les modèles canoniques de diffusion par sauts - décrivant le saut d'un atome dans un double puits de potentiel caractérisé par un temps de résidence moyen fini et une distance de saut finie - ont également été appliqués avec succès dans de nombreux systèmes pour prendre en compte la "granularité" qui produit une déviation de la loi de diffusion Fickienne à grands Q [34]. Dans le Nafion, des données de QENS obtenues en 1980 sur une membrane saturée d'eau ont été analysées avec le modèle de diffusion dans une sphère [35]. Quoique des informations quantitatives sur les échelles de temps et d'espace moyennes, ainsi que le coefficient de diffusion des molécules d'eau confinées, aient été obtenus, les auteurs ont souligné les limitations d'une telle approche. Les spectres sont difficilement ajustés sur toute 
Diffusion translationnelle confinée
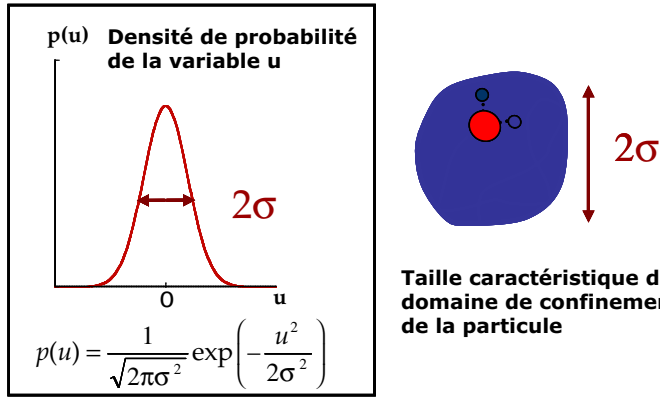

Taille caractéristique du domaine de confinement de la particule

Fonction de diffusion intermédiaire incohérente

$I^{\text {incoh }}(Q, t)=\langle\exp [i Q(u(t)-u(0))]\rangle=\exp \left(-Q^{2} \sigma^{2}[1-\rho(t)]\right)$

Fonction de corrélation

$$
\begin{gathered}
\rho(t)=\frac{\langle u(t) u(0)\rangle}{\sigma^{2}}=\exp \left(-t / \tau_{0}\right)=\exp \left(-D_{\text {eff }} t / \sigma^{2}\right) \\
\text { avec } D_{\text {eff }}=\frac{D_{\text {local }}}{1+D_{\text {local }} Q^{2} \tau_{m i}} \text { et } D_{\text {local }}=\frac{\sigma^{2}}{\tau_{0}}
\end{gathered}
$$

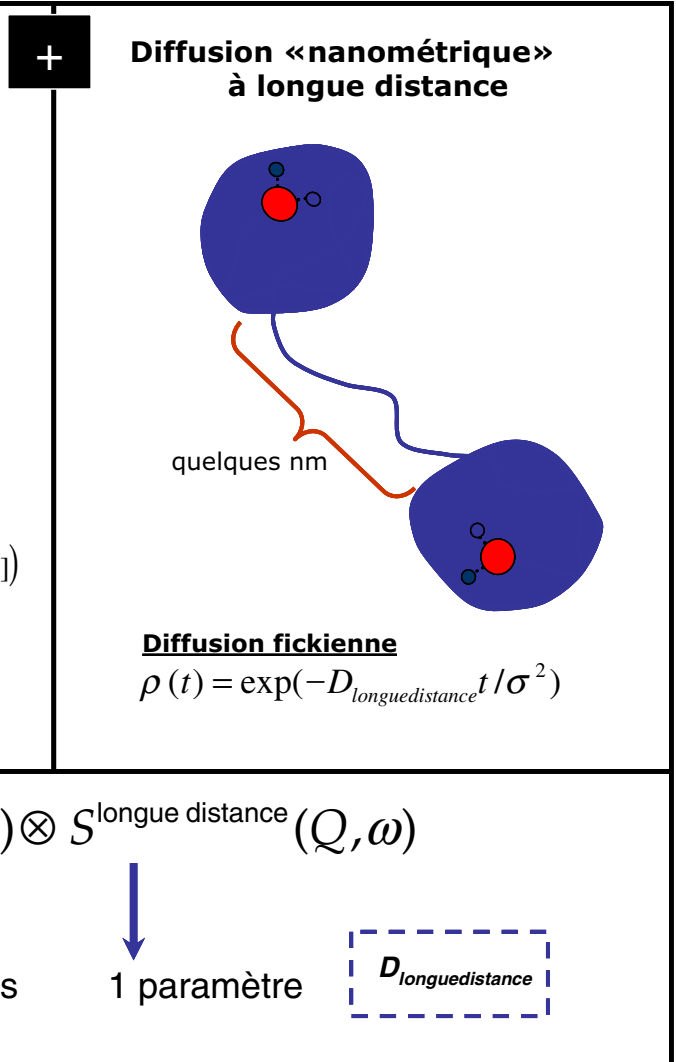

Figure 16. Principe du modèle gaussien (se reporter [37] pour une description détaillée).

la gamme de $\mathrm{Q}$, et la diffusion à longue distance ainsi que les détails microscopiques du mouvement doivent être pris en compte. Il a fallu attendre 2005 pour voir une deuxième étude par QENS consacrée au comportement dynamique de l'eau dans des membranes de Nafion hydraté [36]. La dépendance temporelle et spatiale des mouvements moléculaires a été obtenue via la combinaison de deux modèles, pour lesquels le signal quasiélastique est systématiquement ajusté avec une seule Lorentzienne de largeur $\Gamma$. Le régime à petits $Q$, où la variation presque plate de $\Gamma$ est symptomatique d'un confinement spatial, a été analysé avec le modèle de diffusion dans une sphère, tandis que le régime des grands $Q$ a été analysé avec le modèle de diffusion par sauts de Hall and Ross [28]. Une telle méthode donne évidemment des résultats mais l'analyse à une seule Lorentzienne tend souvent à une simplification peu physique des systèmes réels.

Notre apport a été, dans ce cadre, de porter un soin particulier à l'enregistrement de spectres de QENS dans une gamme étendue de temps de corrélations (de la picoseconde à la nanoseconde), et de développer un modèle théorique unique basé sur une statistique gaussienne [37]. L'hypothèse de départ est que la position du proton est une variable aléatoire gaussienne, caractérisée par sa variance $\sigma^{2}$. Ce modèle remplace avantageusement les modèles usuels de diffusion sur un cercle ou dans une sphère. Il possède plusieurs atouts : i) il n'y a plus de discontinuité inhérente à la condition aux limites imperméables (l'espace de confinement décrit par une variable gaussienne est donc «mou», ce qui correspond bien mieux à la situation physique) ii) l'expression mathématique des fonctions de diffusion est beaucoup plus simple iii) il prend en compte la diffusion translationnelle localisée dans l'espace 

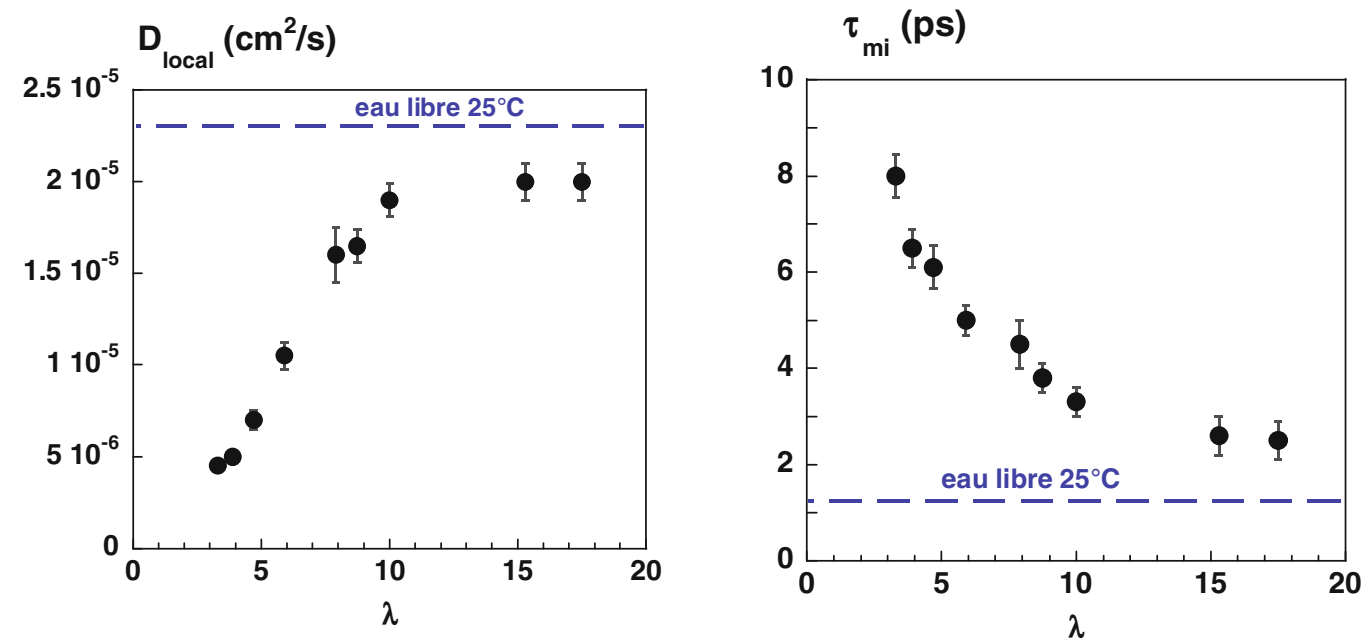

Figure 17. Résultats de l'analyse de spectres quasi-élastiques avec le modèle gaussien. Coefficient de diffusion local $\mathrm{D}_{\text {local }}$ et temps caractéristique $\tau_{\mathrm{mi}}$ en fonction de l'hydratation du Nafion $(\lambda)$.

de confinement, les détails microscopiques du processus élémentaire de saut, et l'existence éventuelle d'une diffusion à longue distance (c'est-à-dire nanométrique).

\subsection{Résultats}

\subsubsection{Dynamique locale}

L'analyse des spectres de diffusion $S(Q, \omega)_{\text {exp }}$ enregistrés sur le spectromètre de temps de vol Mibémol (LLB, Saclay) et de «backscattering » IN16 (ILL, Grenoble) avec le modèle gaussien montre qu'il existe une diffusion translationnelle locale fortement accélérée en fonction de l'hydratation du polymère (Figure 17). Les mesures ont été faites sur des membranes disposées dans des cellules étanches contenant un sel fixant l'humidité relative.

Au-delà d'une dizaine de molécules d'eau par site ionique, le comportement de l'eau est quasiment celui de l'eau liquide. Dans la phase précoce d'hydratation, le coefficient de diffusion local, qui correspond à la diffusion de la molécule d'eau dans une gouttelette au voisinage de la fonction ionique, subit une forte variation.

La variance $\sigma$ qui représente la taille du domaine de confinement peut être comparée à la distance interaggrégats (d) extraite des mesures structurales (déduite de la position du pic ionomère dans les spectres de DNPA). Nos résultats peuvent par ailleurs être comparés avec ceux de la littérature en calculant le diamètre sphérique équivalent $d=2 R=2 \sigma \sqrt{5}$. La Figure 18 compare ainsi nos données $\left(d_{l o c}\right.$ et $\left.d_{L}\right)$ avec celles de Pivovar et al. [36] $\left(d_{\text {piv }}\right)$ et Volino et al. [35] $\left(d_{\text {voli }}\right)$. On observe que, à très faible hydratation, $\sigma$ est légèrement inférieur à l'espace disponible entre agrégats hydrophobes. Dans un premier régime, jusqu'à $\lambda=10$, l'augmentation de la taille moyenne des gouttelettes d'eau adsorbées sur chaque site ionique peut être corrélée au gonflement microscopique. Au-delà de g $\lambda=10$, la corrélation est perdue : tandis qu'il n'y a aucun accident dans l'évolution de $d$, qui est linéaire avec $\lambda$, les distances dynamiques saturent. Le confinement dynamique résulte probablement de l'interaction forte des molécules d'eau dans un cluster d'hydratation dont la taille maximale vaut $2 \sigma_{l o c}$ à $\lambda=10$, soit environ 4 A. Cette interaction gouverne la géométrie des mouvements davantage que le confinement structural. 


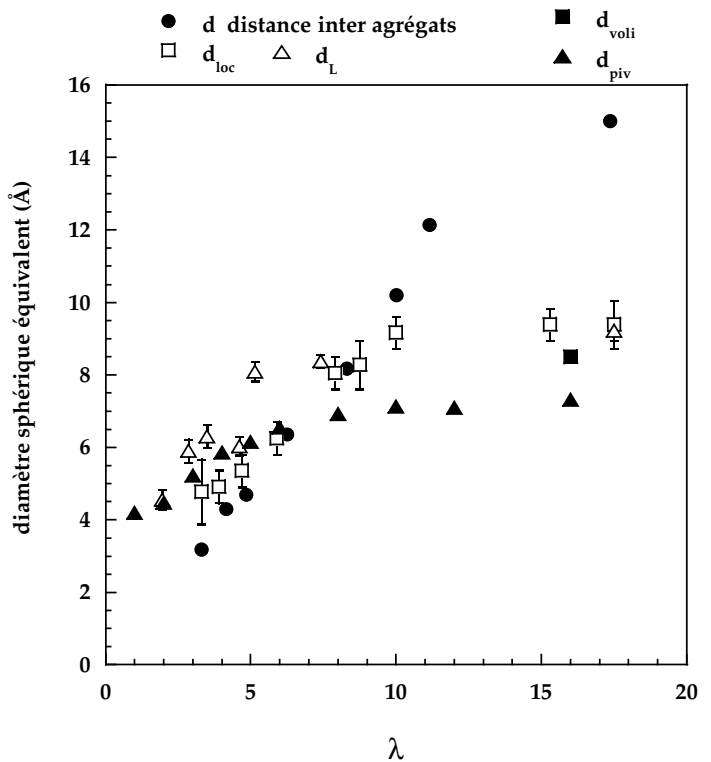

Figure 18. Diamètre sphérique équivalent du domaine de confinement dynamique. Les données de cette étude sont comparées à $\mathrm{d}_{\text {voli }}$ et $\mathrm{d}_{\text {piv }}$, issues respectivement des références 36 et 35 et à la distance structurale interagrégats $d$.

\subsubsection{Dynamique à longue distance}

Le coefficient de diffusion «nanométrique» $\mathrm{D}_{\text {longuedistance varie également fortement avec }}$ l'hydratation. Il peut être comparé aux valeurs de $\mathrm{D}_{s}$, coefficient d'auto-diffusion obtenu par RMN à gradients de champs (Figure 19) et mesuré à une échelle micrométrique.

La figure 19 permet de comparer les trois échelles spatiales sondées : la taille caractéristique d'un cluster d'hydratation (coefficient de diffusion local $D_{\text {local }}$, QENS), la distance entre clusters

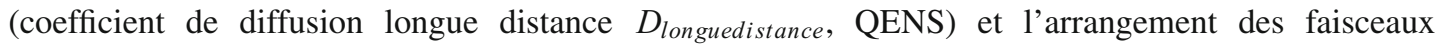
d'agrégats hydrophobes (coefficient de diffusion $D_{S}, \mathrm{RMN}$ ).

Le ralentissement observé entre les différentes échelles spatiales n'est pas uniforme sur toute la gamme d'hydratation étudiée. Il existe deux régimes différents, avec une limite autour de $\lambda=7-10$. Dans la zone des fortes hydratations, à partir de $\lambda=10$, la capacité de sorption du Nafion augmente fortement et les propriétés dynamiques locales de l'eau dans les clusters ioniques sont celles de l'eau bulk $\left(D_{\text {local }}\right)$. Le coefficient de diffusion à l'échelle nanométrique ( $\left.D_{\text {longuedistance }}\right)$ reste du même ordre de grandeur de celui de l'eau bulk (réduction d'un facteur 5). De plus, dans cette zone d'hydratation, $D_{S}$ et $D_{\text {longuedistance }}$ sont égaux et suivent la même loi de variation en fonction du gonflement. Pour les membranes très hydratées, il n'y a donc pas de ralentissement de la diffusion entre l'échelle nanométrique (échelle à laquelle est déterminé $D_{\text {longuedistance) }}$ ) et l'échelle micrométrique (échelle de la mesure de $D_{S}$ ). L'eau ne rencontre pas d'obstacle à sa diffusion entre ces deux échelles spatiales très éloignées : la molécule, une fois qu'elle diffuse d'un cluster ionique à un autre sur une distance typique de quelques nanomètres, diffuse aussi vite d'un agrégat à un autre, d'un faisceau d'agrégat à un autre, ce qui est tout à fait remarquable. La très bonne efficacité de la membrane Nafion gonflée, en termes de conduction protonique, est sans doute due à cette propriété du matériau.

Dans la zone des faibles hydratations, pour $\lambda<10$, la situation est radicalement différente. Les valeurs relatives de $D_{S}$ et $D_{\text {longuedistance }}$ s'écartent l'une de l'autre à mesure que l'on sèche la membrane. Le contraste est saisissant à $\lambda=3$, où le rapport $D_{\text {longuedistance }} / D_{S}$ est maximal et vaut 10 . Un fort ralentissement de la diffusion de l'eau est donc observé entre le nanomètre et le micromètre. Cette perte 


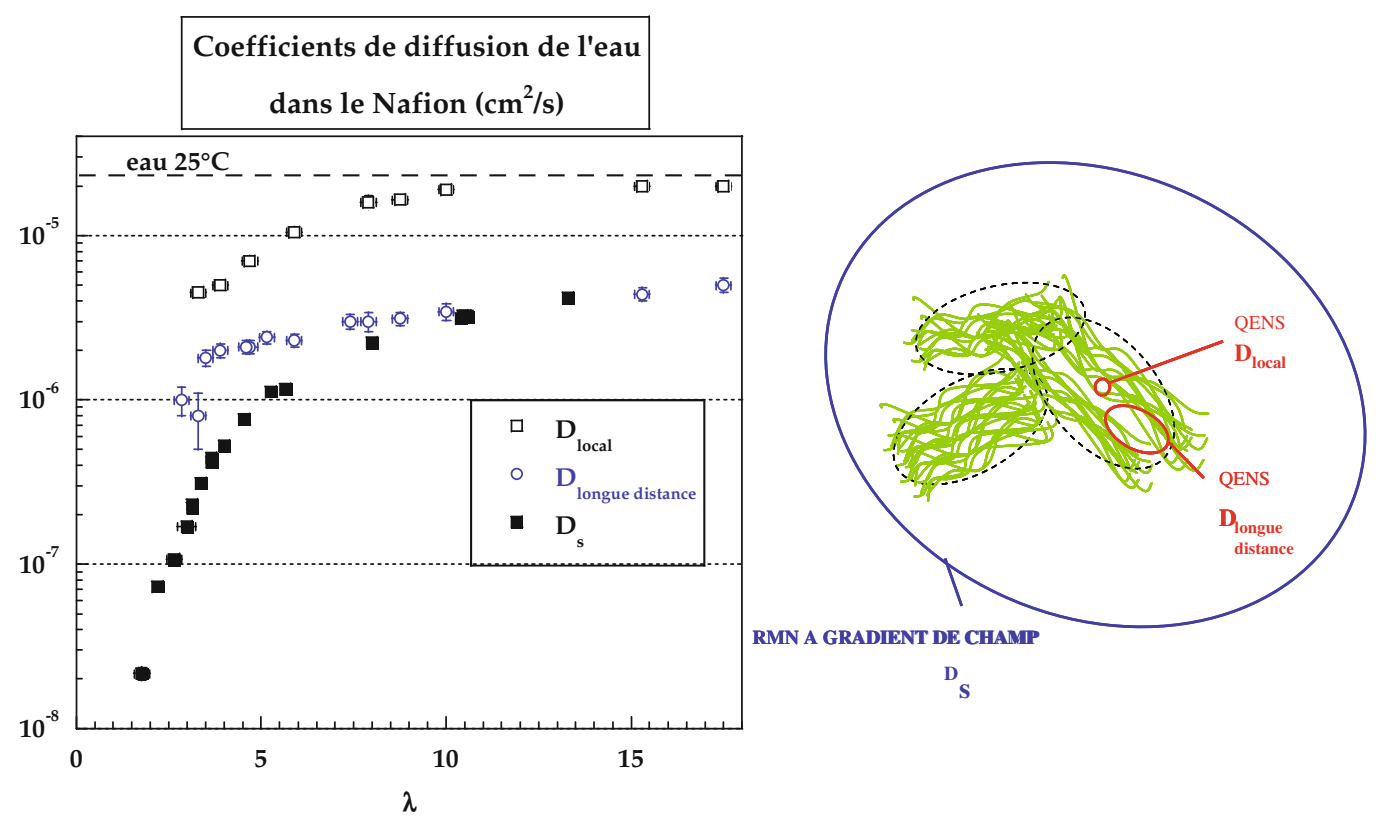

Figure 19. Coefficients de diffusion obtenus dans la membrane Nafion en fonction du degré d'hydratation $\lambda$. Les coefficients $\mathrm{D}_{\text {local }}$ et $\mathrm{D}_{\text {longuedistance }}$ sont tirés des ajustements des spectres quasi-élastiques, le coefficient $\mathrm{D}_{s}$ des mesures par RMN à gradient de champ pulsé.

de mobilité est associée à une diminution notable de la mobilité locale de l'eau dans un cluster ionique $\left(D_{\text {local }}\right)$ et à une diminution importante de la conductivité protonique.

\subsubsection{Apports du QENS : scénario de diffusion moléculaire}

Les expériences de diffusion quasiélastique des neutrons sur des Nafion plus ou moins hydratés permettent in fine de proposer un scénario de la diffusion des protons à l'échelle moléculaire. Trois phases sont identifiées : dans le matériau presque $\sec (\lambda<2)$, les fonctions sulfoniques se dissocient et les ions hydronium sont formés, sans qu'aucune diffusion nanométrique soit mesurable. Ensuite, la phase intermédiaire $(2<\lambda<10)$ est caractérisée par une accélération de tous les paramètres dynamiques. Les molécules d'eau confinées au voisinage des groupements ioniques possèdent une mobilité locale croissante, jusqu'à presque atteindre celle de l'eau liquide. La diffusion d'un cluster d'eau à un cluster voisin est possible et quantifiée par le coefficient de diffusion $\mathrm{D}_{\text {longuedistance, }}$ lequel s'accroît également en fonction de l'hydratation. Aux plus fortes hydratations $(\lambda>10)$, la taille de confinement et la mobilité locale saturent, tandis que la diffusion fickienne continue à s'accélérer.

Ces résultats permettent de reconsidérer la nature des mécanismes de la conduction protonique dans la membrane. La figure 21 montre sur le même graphe l'évolution de la conductivité macroscopique et du coefficient d'auto-diffusion de l'eau mesuré par RMN. La phase de saturation des paramètres de la dynamique moléculaire, pour $\lambda>10$, correspond à une région où les deux grandeurs montrent des variations similaires, ce qui plaide en faveur de l'existence d'un transport principalement de type véhiculaire.

Les informations microscopiques extraites des expériences de QENS sont extrêmement importantes pour alimenter et valider les simulations numériques qui, quoique très nombreuses en ce qui concerne la 


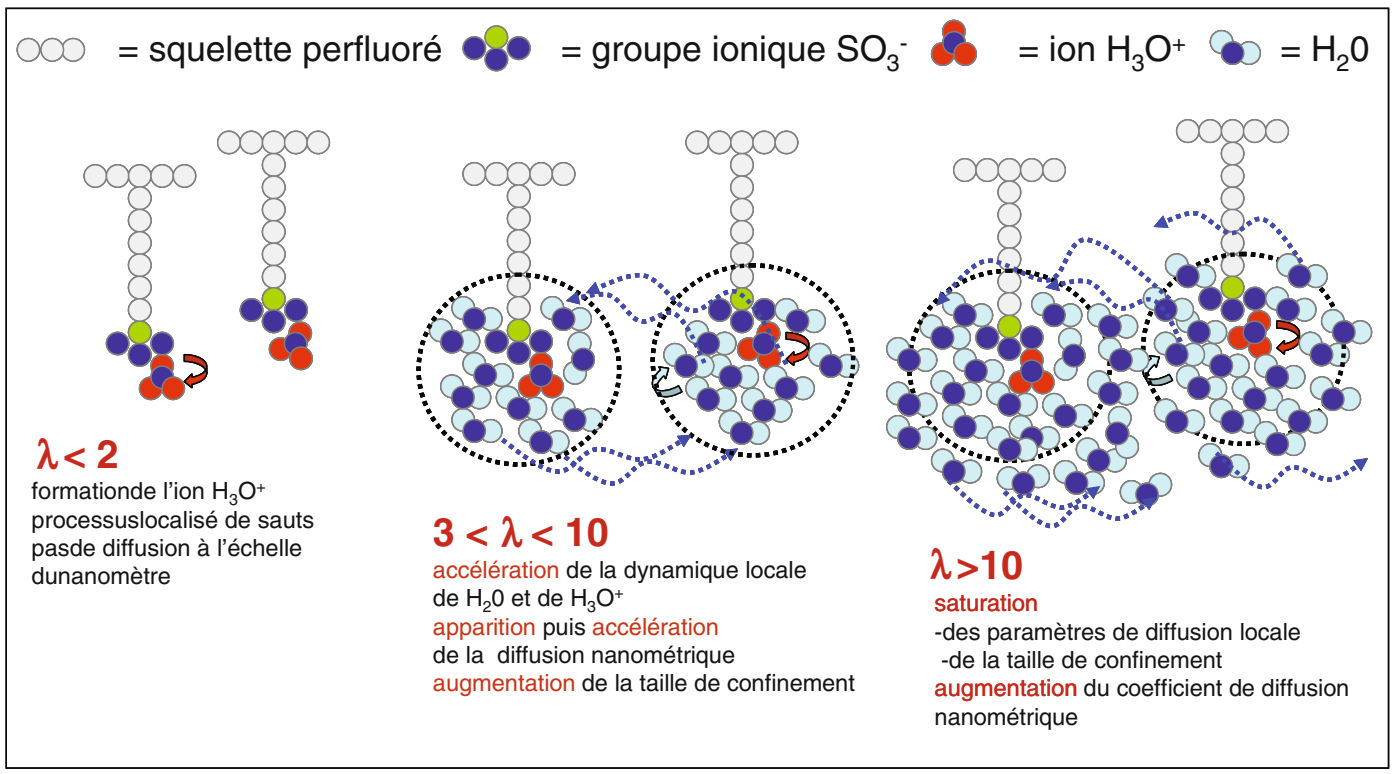

Figure 20. Représentation schématique de la dynamique du proton et des molécules d'eau dans un Nafion en fonction de son hydratation $\lambda$.
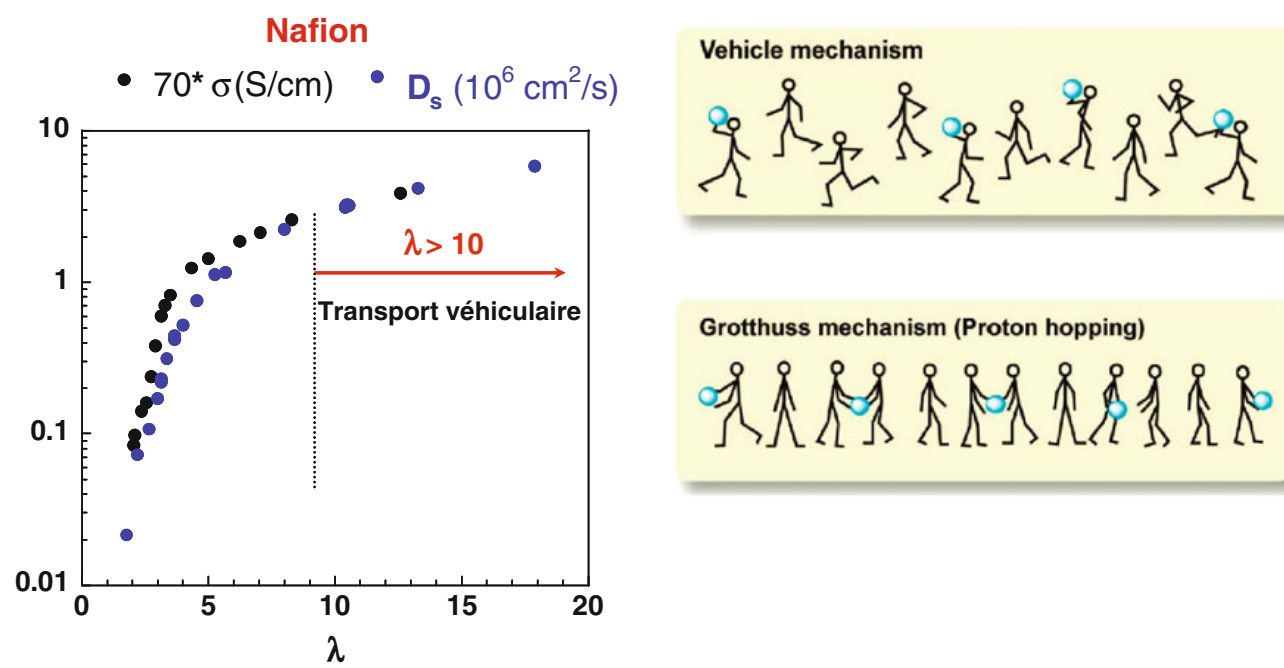

Figure 21. Comparaison de la conductivité protonique (arbitrairement multipliée par 70 pour juxtaposer les données) et du coefficient d'auto-diffusion de l'eau dans le Nafion en fonction de son état d'hydratation $\lambda$.

modélisation structurale, demeurent rares et difficiles dès qu'on aborde les questions dynamiques. Les paramètres que nous avons mesurés sont autant de données injectables dans les modèles de transfert de masse et de charges qui tentent de décrire le transport complexe du proton à travers l'électrolyte dans une pile à combustible. 


\section{Références}

[1] Perfluorinated Ionomer Membranes; American Chemical Society : Washington, 1982; Vol. 180.

[2] Wintersgill, M. C.; Fontanella, J. Complex impedance measurements in Nafion. Electrochim. Acta 1998, 43, 1533.

[3] Kerres, J. A. Development of ionomer membranes for fuel cells. J. Memb. Sci. 2001, 185, 3.

[4] Smitha, B.; Sridhar, S.; Khan, A. A. Solid polymer electrolytes membranes for fuel cell. J. Memb. Sci. 2005, 259, 10.

[5] Byun, H. S.; Burford, R. P.; Fane, A. G. Sulfonation of cross-linked asymmetric membranes based on polystyrene and divinylbenzene J. Appl. Polym. Sci. 1994, 52, 825.

[6] Zschocke, P.; Quellmalz, D. Novel exchange membranes based on aromatic polyethersulfone. J. Memb. Sci. 1985, 325-332.

[7] Alberti, G.; Casciola, M.; Massinelli, L.; Bauer, B. Polymeric proton conducting membranes for medium temperature fuel cells. J. Memb. Sci. 2001, 185, 73.

[8] Marestin C, Gebel G., Diat O., Mercier R. Sulfonated Polyimides, Fuel cells II Advances in Polymer Science (2008), 216, 185-258.

[9] Bahar, B.; Cavalca, S.; Cleghorn, S.; Kolde, J.; Lane, D.; Murthy, M.; Rusch, G. Effective selection and use advance membranes electrodes power assemblies. J. New Mater. Electrochem. Syst. 1999, 2, 179.

[10] Bowen, W. R.; Doneva, T. A.; Yin, H. B. Polysulfone-sulfonated poly(ether ether) ketone blend membranes : systematic synthesis and characterization. J. Memb. Sci. 2001, 181, 253.

[11] Xu F; Diat O., Gebel G. Morin A., Determination of transverse water concentration profile through MEA in a fuel cell using neutron scattering, Journal of the Electrochemical Society (2007), 154 (12), B1389-B1398.

[12] Gebel G., Diat O., Escribano S., Mosdale R. Water profile determination in a running PEMFC by small-angle neutron scattering. Journal of Power Sources, 2008, 179, 132-139.

[13] Mauritz KA and Moore RB. State of understanding of Nafion. Chem. Rev. 2004. 104, 4535-4585.

[14] Rubatat L, Rollet A-L., Gebel G, Diat O. Evidence of Elongated Polymeric Aggregates in Nafion. Macromolecules 2002, 35, 4050-4055.

[15] Rubatat L., Gebel G. Diat O. Fibrillar structure of Nafion : matching Fourier and real space studies of corresponding films and solutions, Macromolecules (2004), 37, 7772-7783.

[16] Kreuer K-D, On the complexity of proton conduction phenomena. Solid States Ionics (2000), 136-137, 149-160.

[17] K.-D. Kreuer, S.J. Paddison, E. Spohr and M. Schuster. Transport in Proton for Fuel-Cell Applications : Simulations, Elementary Reactions, and Phenomenology. Chemical Reviews. 2004, 104, 4637-4678.

[18] Sone Y., Ekdunge P., Simonsson D..J., Proton conductivity of Nafion 117 as measured by a fourelectrode AC impedance method, J. Electrochem. Soc. 143, 1254 (1996).

[19] Zawodzinski T.A., Davey J., Velerio J., Gottesfield S., The water content dependence of electroosmotic drag in proton-conducting polymer electrolytes, Electrochim. Acta 40, 297 (1995).

[20] Kreuer K.D. On the Development of Proton Conducting Polymer Membranes for Hydrogen and Methanol Fuel Cells, J. Memb. Sci. 185, 29 (2001).

[21] Zawodzinski T.A., Neeman M., Sillerud L.O., Gottesfeld S., Determination of water diffusion coefficients in perfluorosulfonate ionomeric membranes, J. Phys. Chem. 95, 6040 (1991).

[22] Gong X., Bandis A., Tao A., Meresi G, Wang Y., Inglefield P.T., Jones A.A., Wen W-Y., Selfdiffusion of water, ethanol and decafluropentane in perfluorosulfonate ionomer by pulse field gradient NMR, Polymer 42, 6485 (2001).

[23] C. Alba-Simionesco et al, Effects of confinement on freezing and melting, J. Phys. Condens. Matter 18 (2006) R15-R68. 
[24] Kreuer K.D., rabenau A., Weppner W, Angew. Vehicle mechanism. A new model for the interpretation of the conductivity of fast proton conductors. Chem. Int. Ed. Ebgl. 21, 208-209 (1982).

[25] Paddison S.J., Reginald P. The nature of proton transport in fully hydrated Nafion. Phys. Chem. Chem. Phys. 4, 1158 (2002).

[26] Leclercq-Hugeux F. et al., Neutrons probing the structure and dynamics of liquids, C.R. Physique 8 (2007) 884-908; Alba-Simionesco C., Etude des propriétés dynamiques des fluides confinés par diffusion quasi-élastique des neutrons, Collection SFN 8 (2007) 43-60.

[27] J-C. Perrin, S. Lyonnard and F. Volino; Quasielastic neutron scattering study of water dynamics in hydrated nafion membranes, Journal of Physical Chemistry C, 111 (2007), 3393-3404.

[28] Hall P.L., Ross D.K., Incoherent Neutron Scattering functions for random jump diffusion in bunded and infinite media. Mol. Phys. 1980, 42, 673.

[29] Dianoux A.J., Volino F., Random motion of a uniaxal rotator in a N-fold cosine potential : correlation functions and incoherent neutron scattering law. Mol. Phys. 1977, 34, 1263.

[30] Dianoux, A.J., Pineri M., Volino F. Neutron incoherent scattering law for restricted diffusion inside a volume with an anisotropic shape - Application to the problem of water in Nafion membranes. Mol. Phys. 1982, 46, 129-137.

[31] Volino F., Dianoux A., Neutron incoherent scattering law for diffusion in a potential of spherical symmetry : general formalism and application to diffusion inside a sphere. Mol. Phys. 1980, 41 (2), 271-279.

[32] Proceedings of the International Conference on Neutron Scattering. Physica B. 1998. Vol. 241243.

[33] Proceedings of the International Workshop on Dynamics in Confinement, European Physical Journal E. 2003.

[34] Teixeira J., Bellissent-funel M-C., Chen S-H., Dianoux A.J. Experimental determination of the nature of diffusive motions of water molecules at low temperatures. Phys. Rev. A. 1985, 31, 1913.

[35] Volino F., Pineri M., Dianoux A.J., De Geyer A. Water mobility in a water-soaked Nafion membrane : a high-resolution neutron quasielastic study. J. Polym. Sci. 1982, 20(3), 481-496.

[36] Pivovar A.M. and Pivovar B.S. Dynamics Behavior of Water within a polymer electrolyte fuel cell membrane at low hydration level. J. Phys. Chem. B (2005), 109, 785-793.

[37] F. Volino, J-C. Perrin and S. Lyonnard; Gaussian model for localized translational motion : application to incoherent neutron scattering, J. Phys. Chem. B, 110 (2006), 11217-11223. 